\title{
Forschungsförderung für die Pädiatrische Onkologie und Hämatologie
}

Die klinische Forschung in der Pädiatrischen Onkologie hat mittels der konsekutiven Therapieoptimierungsstudien [2], die überwiegend von der Deutschen Krebshilfe e.V. und zunehmend von der Deutschen Kinderkrebsstiftung e.V. gefördert werden, zu einem langfristigen Überleben von nahezu $80 \%$ der an Krebs erkrankten Kinder geführt [4]. Aber immer noch sterben zu viele Kinder an Krebs, und die Behandlung hat viele Nebenwirkungen und Folgen. Das erfordert weiterhin höchste Anstrengungen und intensivste Forschung für neue kurative Maßnahmen. Auch kann ein nicht unerheblicher Anteil der Patienten wahrscheinlich mit weniger Therapie gesund werden, als zurzeit verabreicht wird. Hier besteht also ein erheblicher Forschungsbedarf für eine risikoadaptierte Therapie [3].

Die XX. Jahrestagung der Kind-Philipp-Stiftung für Leukämieforschung, deren Abstracts regelmäßig in der Klinischen Pädiatrie veröffentlicht werden (siehe S. 181-198), ist ein geeigneter Anlass, auf die Wichtigkeit der Forschungsförderung hinzuweisen und gleichzeitig für die gewährte Unterstützung zu danken.

Jedes Jahr wird durch die Kind-Philipp-Stiftung vor allem den jungen Forschern in der Pädiatrischen Onkologie und Hämatologie in Wilsede ein Forum geboten, auf dem die Ergebnisse ihrer experimentellen Forschung vorgestellt und unter erfahrener Leitung diskutiert werden können. Der Vergleich der jetzigen Abstracts mit denen der beiden vorhergehenden Tagungen $[5,6]$ lässt die innovativen Forschungsansätze genauso wie die kontinuierliche Ausweitung der bearbeiteten Fragestellungen erkennen. Gleichzeitig wird die vielfältige Forschungsunterstützung erkennbar, die die verschiedenen Arbeitsgruppen durch gemeinnützige Forschungsförderer, z.B. die Deutsche Krebshilfe e.V., die Kinderkrebsstiftung e.V., die Deutsche Leukämie-Forschungshilfe e.V., die Deutsche José Carreras Leukämie-Stiftung u.a. oder lokal tätige Elterninitiativen erfahren.

Die zweite Ebene in der überregionalen Forschungsförderung durch die Kind-Philipp-Stiftung stellen die in unregelmäßigen Abständen stattfindenden Expertentagungen auf der Reisensburg dar. Zu einem Schwerpunktthema der translationalen Forschung werden international führende Wissenschaftler eingeladen, um durch einen Wissens- und Informationsaustausch mit den jeweiligen Experten der Gesellschaft für Pädiatrische Onkologie und Hämatologie deren Aktivitäten voranzubringen; das zurückliegende Symposium hatte als facettenreiches Thema die Immuntherapie onkologischer Erkrankungen zum Inhalt [7].
Mit dem Kind-Philipp-Preis werden dann gleichsam als dritte Ebene der überregionalen Forschungsförderung - umfassende Forschungsergebnisse ausgezeichnet, die die Kenntnisse zur Pathogenese onkologischer bzw. hämatologischer Krankheitsbilder erweitern und damit grundlegende Erkenntnisse für eine vertiefende Diagnostik oder verbesserte Therapie vermitteln [8]. Beispielsweise ist durch einen der beiden letztjährigen Preisträger mit der Entdeckung von KRAS-Mutationen bei Kindern mit Noonan-Syndrom nicht nur die Diagnostik dieser angeborenen Erkrankung verbessert, sondern auch das Verständnis über die Entstehung von Herzfehlern allgemein und speziell bei Leukämien erweitert worden. Der andere Preisträger hat mit seinen molekularbiologischen Untersuchungen in grundlegender Weise die Kenntnis zur Differenzierung von Neuroblastomen mit günstiger und ungünstiger Prognose durch die systematische Evaluation von 40 verschiedenen Genen vertieft. Auch wenn diese Forschungen nur im Rahmen von interdisziplinär arbeitenden Forschergruppen generiert worden sind, kommt dem Engagement des Einzelnen maßgebliche Bedeutung zu, um herausragende Erfolge zu erzielen.

Die Kind-Philipp-Stiftung, die von einer betroffenen Familie ins Leben gerufen wurde, fördert so den Wissenstransfer in hervorragender und konstanter Weise. Hierfür gehört der Familie unser aller Anerkennung und Dank. Der gleiche Dank gebührt aber auch den gemeinnützigen Institutionen, den überregional und regional arbeitenden Elterninitiativen und Privatpersonen, die seit vielen Jahren in selbstloser Weise die Pädiatrische Onkologie und Hämatologie in ihren Forschungen und Behandlungsverfahren unterstützen, sowie den vielen unbekannten Spendern.

Das vorliegende Schwerpunktheft ist mit seinen Originalbeiträgen im Wesentlichen der Verbesserung der Lebensqualität und ihrer Messung bei krebskranken Kindern und Jugendlichen gewidmet. Speziell die Berichterstattung der Arbeitsgemeinschaft Pädiatrische Radioonkologie (APRO) (siehe S. 166-172) und das GPOH-Positionspapier zur Langzeitnachsorge und Spätfolgenerhebung (siehe S. 173-178) sowie das Qualitätshaus als Instrument zur Leistungsverbesserung von Studienzentralen [1] vermitteln den hohen Standard, der innerhalb der Pädiatrischen Onkologie und Hämatologie inzwischen besteht.

Die bisher erreichten Verbesserungen mit einem Langzeitüberleben von fast $80 \%$ [4] rechtfertigen die vielfältigen Förderungen in nachhaltiger 
Weise. Das Erlangen einer der allgemeinen Bevölkerung entsprechenden Lebenserwartung bei vollständiger psychosozialer und beruflicher Integration möglichst aller krebskranken Kinder und Jugendlichen ist und bleibt das erklärte Ziel der interdisziplinär tätigen Behandelnden wie auch der großherzigen Förderer und Spender.

Prof. Dr. H. Jürgens

GPOH-Vorsitzender 1996-2006

Prof. Dr. T. Klingebiel

GPOH-Vorsitzender seit 2006

Prof. Dr. U. Göbel

Bandredakteur

Prof. Dr. R.-D. Kortmann

Bandredakteur

\section{Literatur}

1 Creutzig U, Hannemann J, Krämer I, Zimmermann M, Herold R, Marx FJ. Das Qualitätshaus als Instrument zur Leistungsverbesserung von Studienzentralen in der Pädiatrischen Onkologie und Hämatologie. Klin Pädiatr 2005; 217: 114-119

2 Creutzig U, Henze G, Bielack S, Herold R, Kaatsch P, Klussmann JH, Graf $N$, Reinhardt D, Schrappe M, Zimmermann M, Jürgens H. Krebserkrankungen bei Kindern: Erfolg durch einheitliche Therapiekonzepte seit 25 Jahren. Dt Ärztebl 2003; 100: A842-A852

3 Göbel U, Henze G. Therapieoptimierung, Risikostratifizierung und neue Therapieverfahren. Klin Pädiatr 2006; 218: 129-131

4 Kaatsch P, Blettner M, Spix C, Jürgens H. Das Langzeit-Follow-up in der deutschen pädiatrischen Onkologie als Basis für die Durchführung von Studien mit Langzeitüberlebenden. Klin Pädiatr 2005; 217: 169-176

5 Kabisch H. XVIII. Jahrestagung der Kind-Philipp-Stiftung für Leukämieforschung. Klin Pädiatr 2005; 217: 183-199

6 Kabisch H. XIX. Jahrestagung der Kind-Philipp-Stiftung für Leukämieforschung. Klin Pädiatr 2006; 218: 185-200

7 Klingebiel T, Bader P, Hollatz T, Koehl U, Lehrnbecher T, Meisel R, Dilloo $D$. Immunotherapy in children: Report from the Reisensburg-Symposium October 20-22, 2004 and recent advances. Klin Pädiatr 2006; 218: 355-365

8 Preisträger des Kind-Philipp-Preises 2006. Klin Pädiatr 2006; 218 : 380-381 